\title{
COMPREHENSIVE TOBACCO CONTROL MEASURES - THE OVERVIEW OF THE STRATEGIES RECOMMENDED BY WHO
}

\author{
Adam Fronczak ${ }^{1}$, Kinga Polańska², Bukola Usidame ${ }^{3}$, Dorota Kaleta ${ }^{4}$ \\ ${ }^{1}$ Ministry of Health, Warsaw, Poland \\ ${ }^{2}$ Department of Environmental Epidemiology, Nofer Institute of Occupational Medicine, Łódź, Poland \\ ${ }^{3}$ Department of Public Policy, University of Massachusetts, Boston, USA \\ ${ }^{4}$ Department of Preventive Medicine, Medical University, Łódź, Poland
}

\section{SUMMARY}

According to the World Health Organization, there are more than one billion smokers in the world, and tobacco is said to kill half of its users. The European Region of WHO, with only $15 \%$ of the world's population, faces nearly one third of the worldwide burden of tobacco related diseases. Millions of lives could be saved with effective and comprehensive tobacco control strategy. In response to this, the World Health Organization has offered a wide range of information and recommendations to governments, organizations, health-care professionals, and tobacco users and non-users worldwide.

This paper is focusing on various activities that governments, organizations or even individuals can undertake to help smokers to quit and decrease percentages of tobacco users and save millions of lives.

Key words: tobacco smoking, smoking cessation, treatment of tobacco dependence

Address for correspondence: D. Kaleta, Department of Preventive Medicine, Medical University, Żeligowskiego 7/9, 90-752 Łódź, Poland. Email: dkaleta@op.pl

\section{INTRODUCTION}

Although we know a lot about the health consequences of smoking and the ways to help smokers quit the habit and maintain smoking abstinence, tobacco is still the single most preventable cause of death in the world today. A long-term tobacco user has a $50 \%$ chance of dying prematurely from tobacco-caused disease (1). More than 9 lives are lost every single minute and $70 \%$ of them occur in the developing world (2). Each year, tobacco causes some 5 million premature deaths, with 1 million of these occurring in countries that can least afford the health-care burden. By the year 2030 , tobacco will kill more than 10 million people annually, and claiming more lives than HIV, tuberculosis, maternal mortality, motor vehicle accidents, suicide, and homicide combined $(3,4)$. Halving global cigarette consumption per adult by the year 2020 would prevent about one third of the tobacco-related deaths in 2020 and would almost halve tobacco-related deaths in the second quarter of the century. Such changes could avoid between seven and ten million tobacco-related deaths by 2025 in the European Region. Increasing tobacco cessation in adults is critically important to improving public health in the short to medium term. This is because smokers die from tobacco-related disease in middle age (defined by epidemiologists as 35 to 69 years) as a result of the 25 to 30 -year delay between smoking and the development of serious disease.

Tobacco products are highly addictive. Because tobacco products are carefully designed to undermine efforts to quit using them, quitting is not simply a matter of choice for the majority of tobacco users. Instead, it involves a struggle to overcome an addiction. Many factors combine with tobacco's addictive capacity to make quitting difficult including media depictions and cultural and societal acceptance of tobacco use. Quitting tobacco at any point in life provides both immediate benefits and substantial long-term benefits to health. Effective treatment for tobacco dependence can significantly improve overall public health within only a few years.

Systematic analyses of the smoking cessation interventions for different groups of smokers (such as young people, pregnant women, hospitalized patients, patients with COPD, or with CHD) and evaluation of effectiveness of different types of programmes (such as telephone, mass media interventions, group or individual counselling, workplace interventions, nicotine replacement therapy) are performed by Cochrane Collaboration Group (http:// www.cochrane.org). Such analyses indicate that even minimal assistance can increase cessation rate. Hundreds of controlled scientific studies have demonstrated that treatment can help tobacco users achieve permanent abstinence. People with tobacco dependence should be provided with effective and adequate treatment as in treating other chronic disorders. A minority of tobacco users (whether they smoke or use smokeless products) achieve permanent abstinence in an initial attempt to quit. Most tobacco users who want to quit go through multiple periods of relapse and remission (1). Effective treatment can involve a variety of methods such as a combination of behavioural treatment 
and pharmacotherapy (nicotine replacement and non-nicotine medications). Population-based methods such as telephone help lines and national and international tobacco-free days also can help deliver treatment.

WHO has made the reduction of tobacco use by treatment one of its top disease-control initiatives and has launched a TobaccoFree Initiative (5). The Tobacco Free Initiative (TFI) was established in July 1998 to focus international attention, resources, and action on the global tobacco epidemic. Its objective is to reduce the global burden of disease and death caused by tobacco, thereby protecting present and future generations from the devastating health, social, environmental, and economic consequences of tobacco consumption and exposure to tobacco smoke. In doing this, the initiative provides global policy leadership; encourages mobilization at all levels of society; and promotes the WHO Framework Convention on Tobacco Control (WHO FCTC). It encourages countries to adhere to its principles, and supports them in their efforts to implement tobacco control measures based on its provisions. A lot of effort has been put into the fight against tobacco use. As an example The Bloomberg Initiative launched in 2006 is set up with the sole aim of reducing tobacco use in low and middle-income countries with emphasis on refining and optimizing tobacco control programmes to help smokers stop using tobacco and to prevent children from starting. They also support public sector efforts to pass and enforce key laws and implement effective policies, supporting advocates' efforts to educate communities about the harms of tobacco and to enhance tobacco control activities that work towards a tobacco-free world and to developing a rigorous system to monitor the status of global tobacco use. WHO is playing an important leadership and coordination role in assisting countries with the implementation of effective tobacco control interventions in line with best evidence and policies, while working with other partners in the initiative (6). The WHO Framework Convention on Tobacco Control adopted by the World Health Assembly in 2003 and entered into force in 2005 was developed in response to the globalization of the tobacco epidemic and is an evidence-based treaty that reaffirms the right of all people to the highest standard of health $(7,8)$. It provides the foundation for countries to implement and manage tobacco control. To help make this a reality, WHO introduced the MPOWER Package of measures to provide country level practical assistance for these areas of the FCTC $(3,4)$. As at 2008, the $70 \%$ of the world's population had been covered by selected tobacco control policies: Monitor tobacco use and control policies (34\%); Protect from tobacco smoke (5\%); Offer help to quit tobacco use (8\%); Warn about the dangers of tobacco (8\%); Enforce bans on tobacco advertising, promotion and sponsorship (9\%); Raise taxes on tobacco $(6 \%)$. The treaty has mobilized resources, rallied hundreds of NGOs, encouraged government action, led to political maturation of health ministries, and raised tobacco control awareness in other government ministries and departments.

The European Strategy for Tobacco Control was adopted by the WHO Regional Committee for Europe at its fifty-second session in September 2002 (9). It provides an evidence-based framework and guidance for effective national action and international cooperation. The ESTC recommended that strategic national action should include: implementing age and gender-based promotional and educational programmes aimed at encouraging the cessation of tobacco use; developing and integrating best practices in the treatment of tobacco dependence and prevention of relapse (i.e., behavioural support, counseling services, "quit lines" and routine advice on cessation of tobacco use) into national health programmes, plans and strategies including those for primary health care, alcohol and drugs control, reproductive health, tuberculosis control, etc.; establishing and strengthening programmes of training in smoking cessation techniques for health professionals including physicians, nurses, dentists and pharmacists as well as teachers and community and social workers; establishing in health care facilities programmes for diagnosis, medical advice and treatment of tobacco dependence, with a priority focus on primary health care. It was also suggested that Member States pay particular attention to funding training and cessation services and increase the affordability of treatment for low-income smokers including treatment either at reduced cost or free of charge. Twenty-three Member States reported that they had introduced promotional and educational smoking cessation programmes aimed at encouraging the cessation of tobacco use. Twenty-one Member States are implementing national programmes for prevention, diagnosis and treatment of tobacco dependence as part of primary health care. In 10 additional countries, such programmes are implemented at a subnational level. There are still no Regionwide standards to determine the content and extent of promotional and educational programmes, so effectiveness probably differs widely within the Region.

For low and middle-income countries that do not have the financial resources to support implementation of a comprehensive cessation programme, there are steps that can be taken to help people quit. Smoking bans are effective in treating tobacco dependence, because when the environment is smoke-free, the smoker is encouraged to quit and has greater hope of not smoking again $(3,4,10)$.

This current paper is analysing recommendations for effective tobacco control measures which can be taken at international, national, local, or individual level.

\section{Different Motivations for Taking up, Continuing or Ceasing the Use of Tobacco}

Better health knowledge alone, though crucial, cannot stem the tobacco epidemic, especially because smoking ordinarily starts in adolescence, when long-term risk may be of less concern than peer influences (11). This is also a life phase during which gender identity is firmly established (12). Surveys among American secondary school students found similar smoking rates for girls and boys. But girls who had experienced depression or family violence were more likely to smoke than boys with similar backgrounds (13). Depression is strongly associated with smoking, and women have about twice the rate of depression than men. However, it is not known whether depression is a cause or an effect of smoking, or whether common factors predispose people to both. Studies show girls and women are more likely to fear weight gain than boys, and to initiate and continue smoking for weight control. Recent review articles agreed that women and girls tend to smoke as a "buffer" against negative feelings, while men smoke more from habit or to enhance positive sensations. Some studies among lowincome mothers in Western countries found smoking was used as a "time out" from the demands of caring for young children. Women frequently indicate psychosocial reasons like sensory ef- 
fects and reducing stress as motivations to smoke. Women may be more sensitive to stress than men especially in the premenopausal phase of the menstrual cycle. There is also evidence that women may experience more severe signs of nicotine withdrawal than men. Additionally, women and men respond somewhat differently to nicotine, and female addiction may be reinforced more by the sensory and social context of smoking rather than by nicotine. This may help explain why some studies have found women quit less easily than men; other suggested explanations include lack of social supports, fear of weight gain, the influence of depression often more common among women, and sex-linked differences in hormonal levels (14).

Research suggests that men and boys perceive greater pressure than women and girls to accept the gendered stereotype that men should be rugged, robust and strong (12). Such concepts lead to a dangerous combination of risk-taking and lack of preventive health activities, with relevance for tobacco uptake, quitting and self-care. In many countries, smoking marks the transition to manhood (15, 16), and is deeply embedded in everyday male social relations both business and personal. The findings of the Global Youth Tobacco Survey, the global survey on adolescents aged 13 to 15 and tobacco, suggest that the projections of future tobacco-related deaths world wide might be underestimated because they are based on current patterns of tobacco use among adults, where women are only about one-fourth as likely as men to smoke cigarettes (17). About $24 \%$ of all young smokers started by the age of ten, when they are far too young to understand or resist social expectations (18).

\section{EFFECTIVE TOBACCO CONTROL ACTIVITIES}

\section{Population-based Approach}

Increasing awareness of the dangers involved in smoking will definitely prove a positive effect in increasing the rate of quitting. Raising tobacco taxes, banning advertising and increasing smoke-free places are all strategies to encourage quitting $(3,4)$.

Higher tobacco taxes that lead to higher cigarette prices encourage smokers to quit, reduce the number of cigarettes smoked and prevent initiation among potential new users. According to the World Bank, on average, a price rise of about $10 \%$ on a pack of cigarettes would reduce the demand for cigarettes by about $4 \%$ in high-income countries and by about $8 \%$ in low and middle-income countries. Researchers estimate that half of the effect of a price increase would be on prevalence: a $2 \%$ reduction of prevalence in high-income countries and a $4 \%$ reduction in low and middleincome countries (4). The other half of the reduction would be on the number of cigarettes smoked by those smokers who continued. Youth, minorities, and low-income smokers are two to three times more likely to quit or smoke less in response to price increases. Governments can use tobacco tax revenues to fund quit lines and subsidize clinical cessation services, and providing cessation support may also reduce opposition to other tobacco control policies. Wealthy countries with substantial financial resources should be expected to offer comprehensive quit-smoking services at no or minimal cost, although low and medium-income countries can effectively implement at least some cessation services. Most countries can use lower-cost counselling options effectively, even when medications are beyond budgetary limits.
Tobacco help lines use two approaches: reactive in which smokers can simply telephone the line, and proactive in which counsellors ring callers back and give ongoing telephone support. Proactive telephone counselling can be effective compared to an intervention without personal contact (1). Quit lines have been set up in 29 Member States. Since 2002, Austria, Croatia, Cyprus, the Czech Republic, Kazakhstan, Latvia, Lithuania, Portugal, and Slovakia have established such lines and Armenia and Spain are planning them.

As an example, in the UK there are two free national quit lines operated through the NHS and by an independent organization called Quit. The NHS Stop Smoking Helpline is available 16 hours a day, 7 days a week. Callers are offered counselling on the telephone, are proactively called back or sent e-mails or text messages to provide ongoing support and motivation, and are given details about their local treatment services. There is also the European Network of Quit lines which aims to bring together experienced and newer quit lines across Europe to develop code of practice tools and policy recommendations on smoking cessation. In addition, it aims to ensure that all European member quit lines have access to the best training, advice and support in counseling protocols, evidence-based cessation programmes, technological advice, and web-based interventions $(3,4)$.

Mass media campaigns can increase knowledge about the health effects of smoking and the benefits of stopping, change and reinforce attitudes towards stopping, increase political acceptance of policy measures (such as those on taxation and environmental tobacco smoke), and provide cues to simple action. Mass media campaigns can also promote and support the delivery of and facilitate access to smoking cessation services $(3,4)$.

Community-wide stop-smoking contests such as Quit and Win aimed at motivating smokers to stop have now been carried out in several countries worldwide. In the WHO European region, 33 countries participated in the 2000 campaign. Follow-up studies showed that after one year, on average $20 \%$ of the participants have remained tobacco-free; although the effects on the population including recruitment strategies, abstinence rates, and participation rates varied from country to country (1).

Smoking bans in public places are crucial for protecting the health of non-smokers and smokers $(3,4)$. It gives a clear message that smoking in public places is not socially accepted. Additionally, when the environment is smoke-free, the smoker is encouraged to quit and has greater hope of not smoking again. Unfortunately, only $5 \%$ of the world's population is covered by comprehensive smoke-free laws (3). Twenty-two percent of the 100 biggest cities in the world are completely smoke-free but progress continues. Using the smoke free regulation in Ireland in 2004, bar workers' exposure to second-hand smoke plunged from thirty hours per week to zero $(4,10)$. Among non-smoking adults living in countries with extensive smoke-free law coverage, $12.5 \%$ were exposed to second-hand smoke, compared with $35.1 \%$ with limited coverage, and $45.9 \%$ with no law (10). In many countries, public perceptions shift against smoking, and non-smoking becomes the norm (10). In the United States, various estimates have shown that the development of legislation banning smoking in public places and its strict enforcement could reduce the average consumption of tobacco by between $4 \%$ and $10 \%$ and induce a significant number of smokers to quit (1). 
Although the improvement was achieved regarding elimination of exposure to tobacco smoke in workplaces and public places there is still a problem with such exposure at home and in cars. It is particularly important for the protection of children for whom there are the major places of exposure. Further action on smokefree homes and cars is needed. In particular, health professionals, teachers, and key stakeholders need appropriate training on the issues around smoking in those places and how to address these issues. Additionally, more research to evaluate interventions and develop a more robust evidence base to inform effective action on this issue is needed (19-22).

\section{Intervention to Quit Smoking}

The evidence supports three main categories of intervention: brief opportunistic advice to stop smoking from a health care professional, face-to-face behavioural support and pharmacotherapy, especially nicotine replacement therapy (NRT) and Bupropion. Brief opportunistic advice from a physician is effective and extremely worthwhile from a public health perspective. There is strong evidence that face-to-face behavioural support, individual and in groups, is effective in its own right, as is pharmacotherapy (1). The evidence shows a correlation between the amount of treatment and the success rates achieved (long-term abstinence). Broadly speaking, less intense interventions achieve lower success rates. They usually potentially reach more people $(1,3,4,23)$.

Health professionals have a critical role to play in reducing tobacco use. The systematic analysis of the results of the interventions given by different health professionals is performed by Cochrane Collaboration Group. In one of the analyses pooled data from 17 trials of brief advice given by physician versus no advice (or usual care) detected a significant increase in the rate of quitting ( $R R=1.7$; 95\% CI 1.4-1.9) (23). Additionally, amongst 11 trials where the intervention was judged to be more intensive the estimated effect was higher $(\mathrm{RR}=1.8 ; 95 \%$ CI 1.6-2.1). Direct comparison of intensive versus minimal advice showed a small advantage of intensive advice ( $\mathrm{RR}=1.37$; 95\% CI 1.20-1.56). Direct comparison also suggested a small benefit of follow-up visits. The other analysis performed by Cochrane covered 31 studies comparing a nursing intervention to a control or to usual care (24). The results of this analysis indicate the potential benefits of smoking cessation advice and/or counseling given by nurses to patients, with reasonable evidence that intervention is effective (the intervention significantly increase the likelihood of quitting: $\mathrm{RR}=1.28 ; 95 \% \mathrm{CI} 1.2-1.4)$. The evidence of an effect was weaker when interventions were brief and were provided by nurses whose main role were not health promotion or smoking cessation.

As stated above, even brief and simple advice from health professionals can have a substantial increase on smoking cessation rates. Therefore, one of the strategies to reduce the number of smoking-related deaths is to encourage the involvement of health professionals in counseling for the prevention and cessation of tobacco use. As at 2007, nineteen Member States reported that training in smoking cessation is an integral part of the basic curriculum for medical students, and in even more countries for nursing, dental, and pharmaceutical students. Twenty-five countries reported that they had post-graduate training for doctors in tobacco control and smoking cessation at national level, but there is incomplete information on the content, quality and amount of training that health professionals receive and whether they are informed about evidence-based guidelines on smoking cessation. Six countries referred to offer postgraduate training for doctors at regional level $(3,4)$.

\section{Nicotine Replacement Therapy}

Nicotine Replacement Therapy (NRT) has been added to the 16th WHO Model List of Essential Medicines because of the high-quality evidence of its effectiveness, acceptable safety, and cost-effectiveness $(3,4)$. The analysis performed by Cochrane indicated that all of the commercially available forms of NRT (gum, transdermal patch, nasal spray, inhaler, and sublingual tablets/lozenges) can help people who make a quit attempt to increase their chances to successfully stop smoking (25). NRTs increase the rate of quitting by $50-70 \%$ regardless of setting. The effectiveness of NRT appears to be largely independent of the intensity of additional support provided to the individual.

One basic element for an effective smoking cessation policy is that the treatment of tobacco dependence is an integral part of the national health programme of a country (26). This is reported to be the case in 25 Member States, although no information is available on the extent of treatment programmes. The United Kingdom remains one of the few countries with a comprehensive national programme which is regularly evaluated. The four countries of the UK and Northern Ireland have a national tobacco dependence treatment service that is universally available to all smokers, mainly free of charge through the countries' National Health Service (NHS). In England, NRT is available without prescription through pharmacies and some other stores. It is also available by prescription at a reduced price with some other cessation medications. All prescription medicines including NRT, Bupropion and Varenicline are free to around half of England's population; this is due to the fact that people with low incomes are free from prescription charges. The remaining people pay a small charge for medications.

Half of the Member States for which information is available implemented national programmes for prevention, diagnosis and treatment of tobacco dependence as part of primary health care.

According to available information, products used in nicotine replacement therapy are available over the counter in $42 \mathrm{Member}$ States and Bupropion is available in 36 countries but not available in 7 (mostly Eastern European) countries. Only six countries in the Region (Belgium, Cyprus, Denmark, France, Ireland, and the United Kingdom) partially reimburse these products by their national health care systems, in general limited to those on lower incomes and/or those aged over 65-70 years.

\section{The Cost-effectiveness of Helping Individual Smokers to Overcome Their Dependence}

A 2000 report by the US Surgeon General emphasizes that smoking cessation is one of the most cost-effective of all health care treatments. The data show that smoking cessation is considerably more cost effective than most health care interventions. This means that, in time, introducing smoking cessation into health care systems will release resources for other needs. An analysis of the cost-effectiveness of implementing the 1996 clinical practice guideline on smoking cessation of the US Agency for Health Care 
Policy and Research showed that the cost per quality adjusted life-year saved ranged from US $\$ 1,100$ (£733) to US $\$ 4,500$ ( $£ 3,000)$ versus US $\$ 23,300(£ 15,533)$ for hypertension screening among 40-year-old men and US \$61,700 (£41,133) for annual mammography for 40 to 49 -year-old women. A similar estimate in the United Kingdom showed the cost per additional life-year of introducing a comprehensive system for the treatment of tobacco dependence ranged from $£ 210$ (US $\$ 315$ ) to $£ 870$ (US $\$ 1,300$ ). Again this was compared with a range of 310 medical treatments from an international review, which found an average of US $\$ 25,500$ per life year gained $(1,26)$. Further, comprehensive publicly funded treatment services for smokers have now been in place for over three years in some parts of the United Kingdom, and their actual cost-effectiveness has been estimated. Using conservative assumptions, the cost-effectiveness of the new services was estimated at just over $£ 600$ (US \$960) per life-year gained for treated smokers aged 35-44 years and $£ 750$ (US \$1,200) for those aged 45-54 years. Evaluation of effectiveness in the Russian Federation showed that extensive cessation programmes could increase life expectancy by an average of three years and reduce the risk for early mortality. The World Bank has estimated the cost-effectiveness of publicly funded NRT with $25 \%$ coverage at just under US $\$ 250$ per disability adjusted life-year in Eastern Europe and central Asia and at just under US $\$ 300$ per disabilityadjusted life-year in low and middle-income countries $(3,4)$.

The WHO CHOICE project has been developed to provide a standardized set of methods and tools that can be used to analyse the costs and population health effects of current and possible new interventions to tackle major risk factors. Smoking cessation policies and interventions are cost-effective, and they will save health systems' considerable expenditure by avoiding some of the costs of treating lung cancer and heart disease and other smoking-related diseases in the future. Experience suggests that cost-effectiveness arguments as well as evidence of effectiveness are essential in persuading governments to allocate funding for smoking cessation policies and interventions according to different tobacco control approaches (1).

For cessation, countries should begin with educational campaigns and community-level projects to raise awareness about the risks of smoking. Until this is done, smokers will probably have little demand for help in stopping. Unpaid media and advocacy techniques can be used in addition to paid advertising when resources permit this. These will help inform the public about the dangers of smoking and can act as an umbrella for other cessation activities such as telephone help-lines and Quit and Win competitions (1).

\section{CONCLUSIONS AND RECOMMENDATIONS}

The national picture is influenced by the international one. The tobacco epidemic truly is a global one, meaning that no country can combat it alone. Even countries with advanced regulations and policy cannot risk to loose against multi-national advertising, smuggling and illicit trade with tobacco products. The globalization of the epidemic has forced us to engage internationally to protect existing as well as future generations. It is hoped that in the coming years till 2030, countries will focus on implementation of the FCTC and its protocols. These will include the elimination of tobacco advertising and promotion worldwide, production of vaccine to switch off nicotine receptors, introduction of systematic teaching on tobacco in medical schools globally, packaging of cigarettes in plain black and white wrappers displaying only a brand name and graphic warnings, and economies with a large tobacco farming sector assisted in diversifying crops. It is also hoped that the NRT would be sold over the counter worldwide, introduction of incentives for quitting would include monetary savings through rebates and lower health insurance programmes, funding of tobacco control from a percentage of tobacco tax in most countries, total elimination of duty-free tobacco. In every country, the tax on tobacco would be at least $75 \%$ of the retail price (10). With all these measures put in place, there would be near or total eradication of tobacco use, ultimately saving billions of lives.

Governments should rank treatment as an important publichealth priority. Health-care systems should offer practical interventions to all tobacco users, regardless of economic level, age, and sex. This effort includes preventing and treating tobacco use in children and adolescents, reducing family exposure to tobacco, and providing treatment medications when appropriate. This process is facilitated by incorporating tobacco dependence treatment into drug abuse treatment, reproductive and maternal-child services, and other programmes. Health-care providers should assess and document tobacco use and should provide treatment as an essential part of quality health care. Health-care providers should assume responsibility for learning about tobacco use and treatment, and for providing proven interventions. Providers, educators, and community leaders should take advantage of teachable moments and opportunities for prevention and intervention. Governments and educational systems can help this process by funding treatment and educational programmes for health professionals in training. Governments and health-care organizations should fund treatment based on methods that have been demonstrated to be effective, and should make treatment widely available. Increasing the institutional and human capacity for providing this service involves training health-care workers to deliver treatment, implementing curriculum for students in the health professions, developing resource centers, encouraging the creation and maintenance of centers of excellence in treating tobacco dependence, and reducing the barriers between tobacco users and treatment. Governments, health providers, and community groups share a responsibility for motivating tobacco users to quit and remain abstinent. They should educate the public about the health risks of tobacco use, encourage tobacco users to seek treatment, and help make treatment available, affordable, and accessible. Governments should monitor and report on tobacco use, and should tax and regulate the sale and marketing of tobacco products. These efforts reduce initiation of tobacco use and help fund effective treatments. Responsible regulation of tobacco products reduces tobacco use and limits risk. Regulatory authorities should prohibit marketing strategies that give false reassurances about minimized health risks and divert attention from quitting. Additionally, all possible steps should be taken to reduce the harmfulness of tobacco products. Governments should collaborate to provide meaningful and accurate ratings of nicotine and other chemicals in tobacco products, and to reduce the toxicity and addictiveness of those products. Treatment medications should be at least as accessible as tobacco products. Investing in the science and technology of treatment improves the efficacy 
of treatment for those in diverse populations and under-served groups. Effective treatments should be developed for groups for whom treatment has not been available such as children and adolescents. The FCTC are mobilizing governments worldwide to coordinate their policies in order to effectively deal with the many different requirements of tobacco control using the MPOWER. Universal application of all of these measures is the most effective approach to tobacco treatment. The current escalation in tobacco use and in tobacco-related death and disease can only be reversed by investment in comprehensive tobacco control which includes treatment for tobacco dependence. Governments, health-care and educational systems, community and religious groups as well as news and entertainment media should collaborate in promoting tobacco dependence treatment.

\section{REFERENCES}

1. World Health Organization. WHO European strategy for smoking cessation policy. Geneva: WHO; 2004.

2. de Seixas Correa LF. Media centre: anti-tobacco convention's fifth anniversary. Geneva: WHO; 2010.

3. World Health Organization. WHO report on the global tobacco epidemic, 2008: the MPOWER package. Geneva: WHO; 2008.

4. World Health Organization. WHO report on the global tobacco epidemic, 2009: implementing smoke-free environments. Geneva: WHO; 2009.

5. World Health Organization. Tobacco Free Inniciative (TFI): World Health No Tobacco Day, 31 May 2010 [Internet]. WHO: Geneva; 2010 [cited 2012 Jan 9]. Available from: http://www.who.int/tobacco/wntd/2010/en/.

6. Bloomberg initiative to reduce tobacco use grants program [Internet]. Edinburgh: Tobacco Control Grants; 2009 [cited 2012 Jan 9]. Available from: http://www.tobaccocontrolgrants.org/.

7. World Health Organization. WHO Framework Convention on Tobacco Control. Geneva: WHO; 2003.

8. WHO Framework Convention on Tobacco Control: guidelines for implementation Article 5.3; Article 8; Articles 9 and 10; Article 11; Article 12; Article 13; Article 14 - 2011 edition [Internet]. Geneva: WHO; 2011 [cited 2012 Jan 09]. Available from: http://www.who.int/fctc/protocol/ guidelines/adopted/guidel_2011/en/index.html.

9. World Health Organization. European Strategy for Tobacco Control [Internet]. Copenhagen: WHO Regional Office for Europe; 2002 [cited 2012 Jan 09]. Available from: http://www.euro.who.int/ data/assets/ pdf_file/0016/68101/E77976.pdf.

10. Shafey O, Eriksen M, Ross H, Mackay J. Tobacco atlas. Atlanta, GA: American Cancer Society; 2009.
11. Samet JM, Yoon SY, editors. Women and the tobacco epidemic: challenges for the 21st century [Internet]. Geneva: World Health Organization; 2001 [cited 2012 Jan 09]. Available from: http://whqlibdoc.who.int/hq/2001/ WHO NMH TFI 01.1.pdf.

12. Courtenay WH. Constructions of masculinity and their influence on men's well-being: a theory of gender and health. Soc Sci Med. 2000 May;50(10):1385-401.

13. Simantov E, Schoen C, Klein JD. Health-compromising behaviors: why do adolescents smoke or drink?: identifying underlying risk and protective factors. Arch Pediatr Adolesc Med. 2000 Oct;154(10):1025-33.

14. World Health Organization. Empower women - combating tobacco industry marketing in the WHO European region. Copenhagen: WHO Regional Office for Europe; 2010.

15. Morrow M, Ngoc DH, Hoang TT, Trinh TH. Smoking and young women in Vietnam: the influence of normative gender roles. Soc Sci Med. 2002 Aug;55(4):681-90.

16. Morrow M, Barraclough S. Tobacco control and gender in Southeast Asia. Part I: Malaysia and the Philippines. Health Promot Int. 2003 Sep;18(3):255-64.

17. Global Youth Tobacco Survey Collaborative Group. Tobacco use among youth: a cross country comparison. Tob Control. 2002 Sep;11(3):252-70.

18. Morrow M, Brands A. Gender, health and tobacco. WHO gender and health fact sheets series. Geneva: World Health Organization; 2003.

19. Jarvis MJ, Mindell J, Gilmore A, Feyerabend C, West R. Smoke-free homes in England: prevalence, trends and validation by cotinine in children. Tob Control. 2009 Dec;18(6):491-5.

20. Mills AL, Messer K, Gilpin EA, Pierce JP. The effect of smoke-free homes on adult smoking behavior: a review. Nicotine Tob Res. 2009 Oct;11(10):1131-41.

21. Kabir Z, Manning PJ, Holohan J, Goodman PG, Clancy L. Active smoking and second-hand-smoke exposure at home among Irish children, 19952007. Arch Dis Child. 2010 Jan;95(1):42-5.

22. Ritchie D, Amos A, Phillips R, Cunningham-Burley S, Martin C. Action to achieve smoke-free homes: an exploration of experts' views. BMC Public Health. 2009 Apr 22;9:112.

23. Stead LF, Bergson G, Lancaster T. Physician advice for smoking cessation. Cochrane Database Syst Rev. 2008 Apr 16;(2):CD000165.

24. Rice VH, Stead LF. Nursing interventions for smoking cessation. Cochrane Database Syst Rev. 2008 Jan 23;(1):CD001188.

25. Stead LF, Perera R, Bullen C, Mant D, Lancaster T. Nicotine replacement therapy for smoking cessation. Cochrane Database Syst Rev. 2008 Jan 23;(1):CD000146.

26. Da Costa V. Policy recommendations for smoking cessation and treatment of tobacco dependence. Geneva: World Health Organization; 2003.

Received August 9, 2011 Accepted in revised form January 17, 2012 